$\underset{\text { clinical }}{\text { nephron }}$

Practice
Nephron 2017; 137:57-63

DOI: $10.1159 / 000477271$
Received: March 1, 2017

Accepted after revision: May 3, 2017

Published online: May 18, 2017

\title{
Effect of Age on the Association of Vascular Access Type with Mortality in a Cohort of Incident End-Stage Renal Disease Patients
}

\author{
Tarek Saleh $^{c}$ Keiichi Sumidaa ${ }^{\mathrm{a}-c}$ Miklos Z. Molnar ${ }^{c, g}$ Praveen K. Potukuchic \\ Fridtjof Thomas $^{d}$ Jun Ling Lu ${ }^{c}$ Geeta G. Gyamlani ${ }^{e}$ Elani Streja ${ }^{f}$ \\ Kamyar Kalantar-Zadeh ${ }^{f}$ Csaba P. Kovesdyc, e

\begin{abstract}
${ }^{a}$ Nephrology Center, Toranomon Hospital Kajigaya, Kanagawa, and ${ }^{b}$ Department of Nephrology, Faculty of Medicine, University of Tsukuba, Ibaraki, Japan; Divisions of ${ }^{\mathrm{C} N e p h r o l o g y, ~ D e p a r t m e n t ~ o f ~ M e d i c i n e, ~ a n d ~}{ }^{\mathrm{d} B i o s t a t i s t i c s, ~ D e p a r t m e n t}$ of Preventive Medicine, University of Tennessee Health Science Center, and eNephrology Section, Memphis VA Medical Center, Memphis, TN, and ${ }^{f}$ Harold Simmons Center for Chronic Disease Research and Epidemiology, Division of Nephrology and Hypertension, University of California-Irvine, Orange, CA, USA; 9 Department of Transplantation and Surgery, Semmelweis University, Budapest, Hungary
\end{abstract}

\section{Keywords}

Hemodialysis · Mortality · Chronic kidney disease .

End-stage renal disease $\cdot$ Vascular access

\begin{abstract}
Background/Aims: All hemodialysis (HD) patients are generally recommended to create a fistula first; but to create a mature arteriovenous fistula (AVF) can be challenging in elderly individuals. It is unclear if elderly incident HD patients derive a survival benefit from an AVF over an arteriovenous graft (AVG) or a tunneled central venous catheter (TDC). Methods: We examined the association of vascular access type (AVF, AVG, and TDC with and without a maturing AVF/ AVG at dialysis transition) at HD initiation with all-cause, cardiovascular (CV), and infection-related mortality in 46,786 US veterans using Cox models with adjustment for confounders. Effect modification by age was examined by ex-
\end{abstract}

\section{KARGER}

(C) 2017 S. Karger AG, Basel

E-Mail karger@karger.com

www.karger.com/nef amining associations in pre-specified age subgroups $(<60$, $60-<70,70-<80$, and $\geq 80$ years old), and by including interaction terms. Results: Patients numbering 8,940 (19\%) started HD with an AVF, 1,090 (3\%) with an AVG, 8,262 (18\%) with a TDC and a maturing AVF/AVG and 28,494 (61\%) with a TDC without a maturing AVF/AVG. A total of 13,303 allcause, 4,392 CV, and 1,058 infection-related deaths were observed in the first year after HD transition. Compared to patients with AVF, those with AVG and TDC with and without maturing AVF/AVG had incrementally higher overall risk of all-cause mortality and CV mortality. Only TDC use was associated with higher infection-associated mortality. These associations were not modified by age. Conclusion: Although most of our patients consisted of male veterans and the results may not be generalized to the general population, the use of TDCs is associated with poor outcomes even in the most elderly incident HD patients.

C 2017 S. Karger AG, Basel

Dr. Csaba P. Kovesdy

Nephrology Section, Memphis VA Medical Center

1030 Jefferson Ave.

Memphis, TN 38104 (USA)

E-Mail ckovesdy@uthsc.edu 


\section{Introduction}

The number of elderly patients with end-stage renal disease (ESRD) in the United States has been increasing in recent decades [1]. The National Institute on Aging reports that the United States population age 65 and over is expected to double in size within the next 25 years, and by 2030 more than 70 million people will be 65 years or older and more than 2.24 million patients will have ESRD [1]. Each year, more elderly patients transition to ESRD in the United States, the majority of whom are treated with in-center hemodialysis (HD) and require a vascular access, such as an arteriovenous fistula (AVF) or graft (AVG), or a tunneled central venous catheter (TDC) [2].

The type of vascular access for HD is one of the major factors known to be associated with mortality in ESRD patients. Clinical guidelines, including the Fistula First Breakthrough Initiative, endorse the use of AVF over AVG and TDC because it is associated with better survival and lower complication rates [3-5].

Despite higher initial successful function rates compared with AVFs and lower infection rates compared with TDCs, AVGs are the least used form of vascular access at HD initiation because they tend to require more interventions and are more likely to fail compared with AVFs $[4,6]$. On the other hand, multiple studies have shown that older patients are at greatest risk of having AVF maturation failure, potentially leaving them dependent on TDCs for vascular access [7-9].

With these conflicting results about the different types of vascular access in elderly HD patients, it is unclear if elderly HD patients derive a survival benefit from an AVF over an AVG. Furthermore, knowledge is scarce about the association of access type with outcomes in the oldest HD patients (e.g., $>80$ years old), in whom it is possible that even a TDC could be equivalent to an AVF or AVG. We hypothesized that patients with advanced age may not experience a survival benefit from an AVF over an AVG or a TDC during the immediate post-transition period to $\mathrm{HD}$. Given that placement and maintenance are arguably more invasive for fistulas and grafts, they take a longer time to mature; older individuals may not garner the same benefit from the "fistula-first" approach similar to younger patients who have lower comorbidity levels and longer survival. Therefore, we investigated the association of vascular access type with all-cause and causespecific patient mortality among 4 different age subgroups in a large national cohort of incident HD patients from the US Department of Veterans Administration (VA).

\section{Materials and Methods}

\section{Cohort Definition}

We analyzed data from the Transition of Care in Chronic Kidney Disease study, a retrospective cohort study examining US veterans transitioning to renal replacement therapy from October 1, 2007 through September 30, 2011 [10]. A total of 52,172 US veterans were identified from the US Renal Data System (USRDS) [1] as the initial cohort. We used the vascular access type listed on the USRDS Patient and Medical Evidence Form 2728 to identify patients who initiated HD using an AVF, an AVG, or a TDC with and without a maturing AVF or AVG (treated as separate categories). Patients with missing or unknown information about HD vascular access $(n=4,909)$, those with missing age or age $<18$ years old $(n=7)$, and those with no follow-up information $(n=470)$ were excluded, resulting in a final analytical sample of 46,786 patients. Patients were categorized by their age at HD start into prespecified groups of $<60,60-<70,70-<80$, and $\geq 80$ years old.

\section{Data Collection}

Data from the USRDS Patient and Medical Evidence Form 2728 were used to determine baseline demographic characteristics at the time of dialysis initiation, laboratory variables prior to HD (estimated glomerular filtration rate [eGFR], albumin and hemoglobin), and the presence or absence of Nephrology subspecialty care received prior to HD. Information about comorbidities was extracted from the VA Inpatient and Outpatient Medical SAS Datasets [11] using ICD-9-CM diagnostic and procedure codes and CPT codes, as well as from CMS data files, as previously described [12]. Cardiovascular disease was defined as the presence of diagnostic codes for coronary artery disease, angina, myocardial infarction, or cerebrovascular disease. We calculated the Charlson Comorbidity Index score using the Deyo modification for administrative data sets, without including kidney disease [13].

\section{Statistical Analysis}

Data are presented as a number (percent) for categorical variables and mean \pm SD or median (interquartile range [IQR]) as appropriate. Continuous variables were compared using analysis of variance and categorical variables were compared with chi-square test. The association of various demographic and clinical characteristics with the type of vascular access was examined in multinomial logistic regression analyses.

The co-primary outcomes were all-cause, cardiovascular (CV), and infection-related mortality during the first year after dialysis initiation. Information about all-cause mortality was obtained from the VA Vital Status Files [14], and causes of death were obtained from the USRDS. We examined the association of vascular access type with outcomes during the first year following HD initiation using the Kaplan-Meier method and the log-rank test. Hazard ratios (HRs) of all-cause mortality were calculated in unadjusted and multivariable adjusted Cox models. All multivariable models were adjusted based on a priori considerations for age, gender, race, comorbid conditions (history of diabetes mellitus, myocardial infarction, congestive heart failure, peripheral vascular disease, cerebrovascular disease, dementia, chronic lung disease, liver disease, and malignancies), and for the Charlson comorbidity index as an omnibus measure of illness. The effect of age on the association of vascular access type with the various outcomes was additionally examined by performing analyses in subgroups of pa-
58

Nephron 2017;137:57-63

DOI: $10.1159 / 000477271$
Saleh et al. 
Table 1. Baseline characteristics of patients categorized according to the type of vascular access used to initiate hemodialysis

\begin{tabular}{|c|c|c|c|c|c|}
\hline & $\begin{array}{l}\text { AVF } \\
(n=8,940)\end{array}$ & $\begin{array}{l}\text { AVG } \\
(n=1,090)\end{array}$ & $\begin{array}{l}\text { TDC, maturing } \\
\text { AVF/AVG } \\
(n=8,262)\end{array}$ & $\begin{array}{l}\text { TDC, no maturing } \\
\text { AVF/AVG } \\
(n=28,494)\end{array}$ & $p$ value \\
\hline Age, years & $70.2 \pm 11.5$ & $70.7 \pm 11.9$ & $70.3 \pm 11.7$ & $71.0 \pm 12.0$ & $<0.001$ \\
\hline Gender, male & $8,582(96)$ & $1,121(91)$ & $7,797(94)$ & $26,903(94)$ & $<0.001$ \\
\hline Race (black) & $2,148(24)$ & $462(37)$ & $2,073(25)$ & $6,814(24)$ & $<0.001$ \\
\hline Ethnicity (non-hispanic) & $8,348(93)$ & $1,168(95)$ & $7,746(94)$ & $28,861(94)$ & 0.009 \\
\hline Pre-dialysis nephrology care & $8,187(96)$ & $1,020(87)$ & $6,101(81)$ & $13,596(56)$ & $<0.001$ \\
\hline Diabetes mellitus & $5,277(66)$ & $743(67)$ & $5,138(71)$ & $16,308(64)$ & $<0.001$ \\
\hline Myocardial infarction & $1,937(24)$ & $284(26)$ & $2,150(30)$ & $7,964(31)$ & $<0.001$ \\
\hline Congestive heart failure & $3,984(50)$ & $577(52)$ & $4,406(61)$ & $15,596(61)$ & $<0.001$ \\
\hline Peripheral vascular disease & $3,102(39)$ & $441(40)$ & $3,079(42)$ & $10,327(41)$ & 0.004 \\
\hline Cerebrovascular disease & $2,360(30)$ & $378(34)$ & $2,348(32)$ & $8,219(32)$ & $<0.001$ \\
\hline Dementia & $160(2)$ & $60(5)$ & $197(3)$ & $850(3)$ & $<0.001$ \\
\hline Chronic lung disease & $3,079(39)$ & $470(43)$ & $3,282(45)$ & $12,137(48)$ & $<0.001$ \\
\hline Liver disease & $819(10)$ & $138(13)$ & $819(11)$ & $3,199(13)$ & $<0.001$ \\
\hline Malignancy & $1,965(25)$ & $291(26)$ & $1,749(24)$ & $6,803(27)$ & $<0.001$ \\
\hline $\begin{array}{l}\text { Last estimated GFR prior to dialysis, } \\
\qquad \mathrm{mL} / \mathrm{min} / 1.73 \mathrm{~m}^{2}\end{array}$ & $11.5 \pm 4.14$ & $12.0 \pm 4.4$ & $11.7 \pm 4.7$ & $12.4 \pm 5.4$ & $<0.001$ \\
\hline Charlson comorbidity index & $4.4 \pm 2.8$ & $4.9 \pm 3.1$ & $4.9 \pm 2.8$ & $4.9 \pm 3.0$ & $<0.001$ \\
\hline Last serum albumin prior to dialysis, g/dL & $3.4 \pm 0.60$ & $3.3 \pm 0.63$ & $3.2 \pm 0.63$ & $3.0 \pm 0.67$ & $<0.001$ \\
\hline Last hemoglobin prior to dialysis, g/dL & $10.3 \pm 1.5$ & $10.1 \pm 1.5$ & $9.9 \pm 1.5$ & $9.9 \pm 1.6$ & $<0.001$ \\
\hline
\end{tabular}

Results presented as means \pm SDs and number (percent).

AVF, arteriovenous fistula; AVG, arteriovenous graft; TDC, tunneled central venous catheter.

tients categorized by their baseline age, and by including multiplicative interaction terms.

Of the variables included in the main multivariable model, data points were missing for race $(<0.1 \%)$ and for comorbid conditions (11\%). Complete data for multivariable analysis was available for 41,578 patients ( $89 \%$ ); due to the relatively low proportion of missingness, missing data was not imputed.

In sensitivity analyses, we examined the same associations over the first 6-month post-transition time period, and then repeated the examination after making additional adjustments for the last eGFR, hemoglobin and serum albumin levels before transition to HD (obtained from USRDS Form 2728). Analyses were conducted using STATA MP version 14 (STATA Corporation, College Station, TX, USA).

\section{Results}

Overall, patients were $70 \pm 12$ years old, 94\% were male, 25\% were African-American, and 58\% had diabetes. Patients numbering 8,940 (19\%) started HD with an AVF; 1,090 (2.3\%) with an AVG; 8,262 (18\%) with a TDC and a maturing AVF or AVG indicating prior attempts to create a permanent vascular access (of which 7,549 had a maturing AVF and 713 had a maturing AVG); and 28,494 (61\%) with a TDC and no maturing AVF or AVG (indicating no attempts to create a perma-

Vascular Access and Mortality in Incident ESRD nent vascular access in the pre-dialysis time period). $\mathrm{Pa}$ tients' baseline characteristics at the time of initiating dialysis by their initial vascular access type are presented in Table 1. Compared to patients with a mature AVF, patients with an AVG were more likely to be female and black, and had not received Nephrology care during the pre-dialysis period. Compared to patients with a mature AVF, patients with a TDC and no maturing AVF/AVG had a higher prevalence of $\mathrm{CV}$ and chronic lung disease and were substantially less likely to have received $\mathrm{Ne}$ phrology care during prelude. Patients with a TDC who had a maturing AVF/AVG had similar or fewer comorbidities as those with a TDC and no maturing AVF/ AVG, with the exception of diabetes mellitus, which was more common in the former group. Furthermore, the group with TDC and maturing AVF/AVG had a higher likelihood of receiving pre-dialysis nephrology care compared to those with a TDC and no maturing AVF/AVG (although it was still lower when compared to the group with a mature AVF).

During the first year after transition to $\mathrm{HD}, 13,303$ patients died (mortality rate 343/1,000 patient-years, 95\% CI 338-350). Figure 1 shows cumulative unadjusted event curves for overall all-cause (Fig. 1a), CV (Fig. 1b), and infection-related (Fig. 1c) mortality in pa-

Nephron 2017;137:57-63 DOI: $10.1159 / 000477271$ 


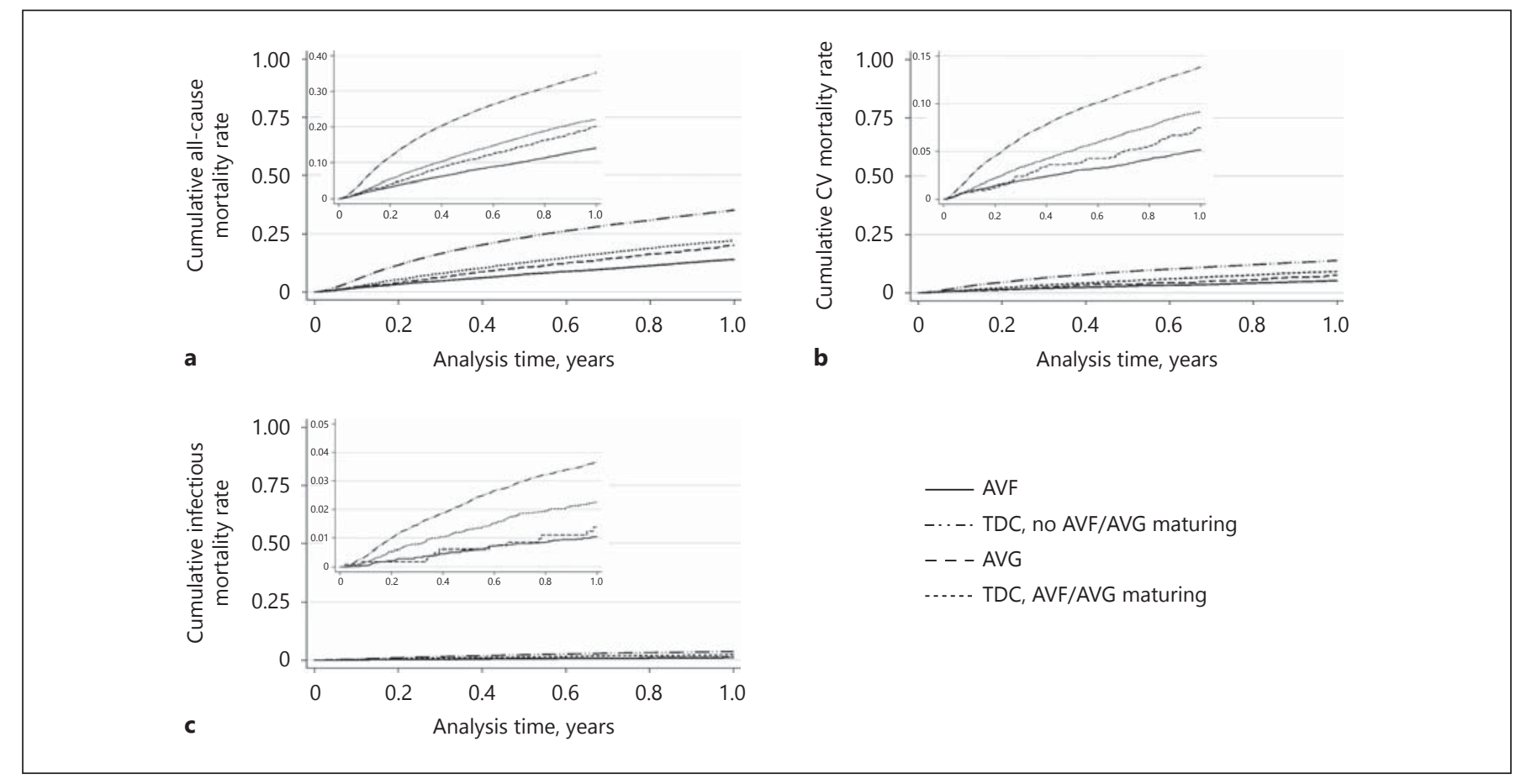

Fig. 1. Cumulative event curves for overall all-cause (a), cardiovascular (b), and infection-related (c) mortality in patients categorized on the basis of their initial vascular access type.

tients grouped by their initial vascular access type, indicating incrementally higher risk of all-cause and CV mortality in patients with an AVG, a TDC and maturing AVF/AVG, and a TDC without maturing AVF/AVG, compared to patients with a mature AVF. Table 2 shows the crude and multivariable adjusted HRs of all-cause, $\mathrm{CV}$, and infection-related mortality associated with AVG and TDC (compared to AVF) in patient subgroups of various ages. All-cause mortality trended higher in patients with an AVG vs. AVF in all age groups; patients with a TDC and a maturing AVF/AVG experienced higher mortality than those with a mature AVF or AVG; and patients with a TDC and no maturing AVF/AVG experienced the worst mortality of all 4 groups in all age categories. The association of vascular access type with all-cause mortality was not significantly modified by age ( $p$ value for interaction $=0.08$ ). The associations of vascular access type with CV mortality were similar in nature and magnitude to those seen for all-cause mortality, with incremental risk associated with AVG, TDC with and without maturing AVF/AVG, and with no effect modification by age ( $p$ value for interaction $=0.40$; Table 2). Compared to patients with AVF, the risk of infection-related mortality was not significantly higher in patients with AVG, although the number of events pre- cluded assessment in some age groups. The risk of infection-related mortality was highest in patients with TDC and no maturing AVF/AVG (multivariable adjusted HRs ranging from 2.07 to 8.92 in various age subgroups), followed by those with TDC and maturing AVF/AVG (multivariable adjusted HRs ranging from 1.09 to 4.28 in various age subgroups), without significant effect modification by age ( $p$ value for interaction $=0.45$; Table 2 ).

Results remained unchanged in sensitivity analyses examining outcomes occurring over the first 6 months following HD transition, and after additional adjustments for pre-transition eGFR, hemoglobin, and serum albumin (data not shown).

\section{Discussion}

In this large and nationally representative retrospective cohort study of incident HD patients, we compared the association of AVG and TDC (vs. AVF) with all-cause and cause-specific mortality in US veterans aged $<60,60$ $<70,70-<80$, and $\geq 80$ years old. We found that using a TDC is associated with higher mortality in all age groups, the risk being the highest for infection-related mortality. Patients with a TDC and no maturing AVF/AVG had 
higher mortality rates compared to patients with a TDC and a maturing AVF/AVG, but the latter had worse outcomes compared to patients with a mature AVF or AVG. Even though the risk of various outcomes associated with AVG vs. AVF was not statistically and significantly different in all age groups, the use of an AVG was associated with an overall modestly increased risk of all-cause and CV mortality, with no effect modification by age upon statistical testing.

Older age is often considered a challenge to the creation and use of vascular access. Given the complicated process of establishing a successful vascular access, clinicians are faced with difficult decisions regarding the choice of an optimal access in elderly CKD patients transitioning to $\mathrm{HD}$, who face a high chance of having an access created but not used (either due to succumbing to death prior to reaching ESRD or due to primary maturation failure) $[15,16]$. Recent studies showed a high rate of primary maturation failure in native AVFs $[15,17]$, yet there are no standard patient eligibility criteria to guide AVF placement. Currently, the primary and secondary fistula patency rate in the elderly at 1 year have ranged from 43 to $74 \%$ and $56-82 \%$, respectively [15]. Older age has been associated with lower rates of fistula use, which is attributable to a decrease in referral for AVFs as well as increased rates of failure to mature among those who do get a fistula $[5,8,17]$.

On the other hand, AVGs are the least used form of vascular access at HD initiation, presumably due to the lower long-term patency rates and an increased association with morbidity and mortality $[4,6]$. The cumulative primary patency at 1 and 2 years was 81 and $65 \%$, respectively, in a study of 67 elderly patients over the age of 70 years, and the secondary patency was 65 and $58 \%$ at 1 and 2 years, respectively [18]. In another study, the 1- and 2 -year total patency rate among elderly patients with synthetic grafts was 44.2 and $38.6 \%$, respectively [19]. However, AVGs are considered viable options in patients with failed fistulas, exhausted, unsuitable, or damaged veins, or when there is late nephrology referral and need for urgent cannulation with avoidance of central venous catheters [20]. Overall, AVF survival is no better than AVG when primary failures are included in access-survival analyses [20,21]. In a study comparing access survival by access type among those aged $>65$ years old using data from the USRDS [22], use of an AVF vs. AVG was not associated with increased patency among non-diabetic (OR $1.48,95 \%$ CI $0.95-2.3$ ) or diabetic elderly (OR 1.49, 95\% CI 0.76-2.89). Placing an AVG first may in fact dramatically lengthen the proportion of the patient's lifespan

Vascular Access and Mortality in Incident ESRD

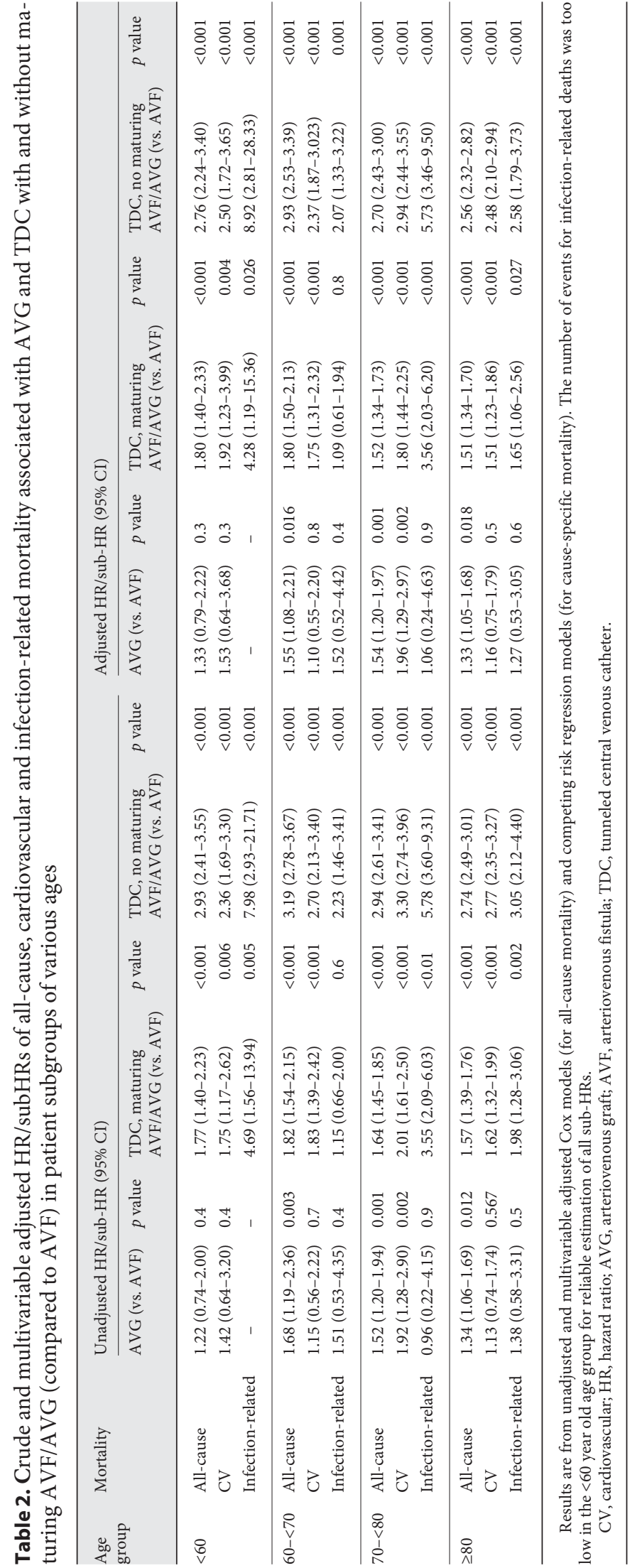

Nephron 2017;137:57-63 DOI: $10.1159 / 000477271$ 
with freedom from catheter dependence and its potential complications [20].

Similar to our study, in a previous observational study of 66,595 elderly ( $>67$ years old) Medicare USRDS patients, the use of AVF as initial access for long-term dialysis therapy was associated with the best survival, followed by AVG and TDC use [22]. In another large analysis of patients $>67$ years old from USRDS, incident catheter use was associated with a significantly higher long-term mortality compared to AVF use, but the association of AVG use with mortality was significant only in relatively younger patients ( $<80$ years old) and was not present in patients $\geq 80$ years old [23]. The seemingly contradictory results regarding the association of AVG with mortality in these studies may be caused by differences in sample size and analytical approaches, as the point estimates for the risk were fairly modest and similar in magnitude in these 2 studies $[4,23]$. Our study supports and extends these findings by examining cause-specific mortality and emphasizing the major importance of infection-related deaths for TDC use, and by including a full spectrum of patient age and thus establishing that age is not a significant effect modifier of the associations between access type and mortality.

In our study, TDC use was associated with the worst outcomes even when accounting for pre-dialysis attempts to create a permanent vascular access (AVF or AVG), although patients with such attempts experienced better outcomes compared to patients with no prior attempts. A recent study examining 2,300 incident HD patients from 5 Canadian dialysis centers also found that patients who do not undergo attempts to place pre-dialysis AVF had worse outcomes compared to those who had such attempts, independent of age, and suggested that the mortality associated with TDC use may not be caused by catheters but by the underlying comorbid conditions necessitating catheter placement [24].

These results are difficult to compare with our findings due to marked differences in sample size, in the nature of the 2 study populations (Canadian vs. US), and the different analytical approaches (we examined immediate postdialysis mortality, as opposed to longer-term mortality in the study by Quinn et al. [24]). However, the fact that in our study even patients with TDC who had attempts at creating an AVF or AVG experienced significantly worse outcomes compared to patients with AVF, and the fact that associations were particularly strong with infection-related mortality suggest that a direct effect of catheter use on adverse outcomes cannot be ruled out. Findings similar to those of our study were also reported recently by Brown et al. [25], who showed that patients initiating HD with a TDC after failed AVF placement had significantly lower all-cause mortality rates than the TDC-only group but higher mortality than patients initiating dialysis using an AVF.

Our study is notable for its large sample size of latestage, non-dialysis-dependent CKD patients transitioning to dialysis for representing the full age spectrum of such patients, and for being representative of veterans in the entire geographic United States. However, several limitations need to be acknowledged. This study was observational, and therefore susceptible to residual confounding and confounding by indication. Even though we adjusted for numerous available confounders, the possibility of residual confounding remains. This may be especially important in the case of patients who transitioned to dialysis using a TDC who have no maturing AVF/ AVG, as catheters are the sole form of vascular access in patients who need emergent initiation of renal replacement therapy, a group that is known to experience worse outcomes [26]. Most of our patients consisted of male veterans; therefore, the results may not be generalized to other cohorts or the general population. We used administrative data for our study, which could be prone to a sampling bias and inaccurate measurement of the predictor variables.

In conclusion, an AVF at $\mathrm{HD}$ transition is associated with lower all-cause, $\mathrm{CV}$, and infection-related mortality across all age categories, including even the oldest incident HD patients. Using a TDC is associated with higher mortality in all age groups, even in patients with prior attempts to creating an AVF or an AVG. Our study thus suggests that a mature AVF at the time of $\mathrm{HD}$ transition is the preferable access even in all incident HD and the most elderly patients. Further studies are needed to obtain more information related to this field about the best strategies to adopt in order to increase AVF placement and maturation in those with advanced age. AVGs may offer an acceptable, and in some cases, even a desirable alternative to AVFs in elderly individuals, due to their more predictable and shorter maturation, thus minimizing the duration of TDC exposure and potentially decreasing the potential risks associated with the latter.

\section{Acknowledgments}

This study is supported by grant 5U01DK102163 from the National Institute of Health to K.K.-Z. and C.P.K., and by resources from the US Department of Veterans Affairs.

The data reported here have been supplied by the USRDS. Support for VA/CMS data is provided by the Department of Veterans
Saleh et al. 
Affairs, Veterans Health Administration, Office of Research and Development, Health Services Research and Development, VA Information Resource Center (project numbers SDR 02-237 and 98-004).

C.P.K. and K.K.-Z. are employees of the Department of Veterans affairs. The interpretation and reporting of these data are the responsibility of the authors and in no way should be seen as official policy or interpretation of the Department of Veterans Affairs or the US government. The results of this paper have not been published previously in whole or part.

\section{Statement of Ethics}

The study was approved by the Institutional Review Boards of the Memphis and Long Beach VA Medical Centers, with exemption from obtaining informed consent.

\section{Disclosure Statement}

The authors have no conflicts of interest to declare.

\section{References}

1 Saran R, Li Y, Robinson B, Abbott KC, Agodoa LY, Ayanian J, Bragg-Gresham J, Balkrishnan R, Chen JL, Cope E, Eggers PW, Gillen D, Gipson D, Hailpern SM, Hall YN, He K, Herman W, Heung M, Hirth RA, Hutton D, Jacobsen SJ, Kalantar-Zadeh K, Kovesdy CP, Lu Y, Molnar MZ, Morgenstern H, Nallamothu B, Nguyen DV, O'Hare AM, Plattner B, Pisoni R, Port FK, Rao P, Rhee CM, Sakhuja A, Schaubel DE, Selewski DT, Shahinian V, Sim JJ, Song P, Streja E, Kurella Tamura M, Tentori F, White S, Woodside K, Hirth RA: US renal data system 2015 annual data report: epidemiology of kidney disease in the United States. Am J Kidney Dis 2016;67(3 suppl 1):Svii, S1-S305.

2 Vascular Access 2006 Work Group: Clinical practice guidelines for vascular access. Am J Kidney Dis 2006;48(suppl 1):S176-S247.

3 Lacson E Jr, Wang W, Hakim RM, Teng M, Lazarus JM: Associates of mortality and hospitalization in hemodialysis: potentially actionable laboratory variables and vascular access. Am J Kidney Dis 2009;53:79-90.

4 Xue JL, Dahl D, Ebben JP, Collins AJ: The association of initial hemodialysis access type with mortality outcomes in elderly Medicare ESRD patients. Am J Kidney Dis 2003;42: 1013-1019.

5 Pisoni RL, Arrington CJ, Albert JM, Ethier J, Kimata N, Krishnan M, Rayner HC, Saito A, Sands JJ, Saran R, Gillespie B, Wolfe RA, Port FK: Facility hemodialysis vascular access use and mortality in countries participating in DOPPS: an instrumental variable analysis. Am J Kidney Dis 2009;53:475-491.

6 Lok CE, Sontrop JM, Tomlinson G, Rajan D, Cattral M, Oreopoulos G, Harris J, Moist L: Cumulative patency of contemporary fistulas versus grafts (2000-2010). Clin J Am Soc Nephrol 2013;8:810-818.

7 Monroy-Cuadros M, Yilmaz S, Salazar-Banuelos A, Doig C: Risk factors associated with patency loss of hemodialysis vascular access within 6 months. Clin J Am Soc Nephrol 2010;5:1787-1792.

8 Lok CE, Allon M, Moist L, Oliver MJ, Shah H, Zimmerman D: Risk equation determining unsuccessful cannulation events and failure to maturation in arteriovenous fistulas (REDUCE FTM I). J Am Soc Nephrol 2006; 17:3204-3212.

9 Miller CD, Robbin ML, Allon M: Gender differences in outcomes of arteriovenous fistulas in hemodialysis patients. Kidney Int 2003;63: 346-352.

10 Sumida K, Molnar MZ, Potukuchi PK, Thomas F, Lu JL, Jing J, Ravel VA, Soohoo M, Rhee CM, Streja E, Kalantar-Zadeh K, Kovesdy CP: Association of slopes of estimated glomerular filtration rate with post-end-stage renal disease mortality in patients with advanced chronic kidney disease transitioning to dialysis. Mayo Clin Proc 2016;91:196-207.

11 VIReC Research User Guide: VHA Medical SAS Inpatient Datasets FY2006-2007. Hines, US Department of Veterans Affairs VA Information Resource Center, 2007.

12 Kovesdy CP, Norris KC, Boulware LE, Lu JL, Ma JZ, Streja E, Molnar MZ, Kalantar-Zadeh K: Association of race with mortality and cardiovascular events in a large cohort of US veterans. Circulation 2015;132:15381548.

13 Deyo RA, Cherkin DC, Ciol MA: Adapting a clinical comorbidity index for use with ICD-9-CM administrative databases. J Clin Epidemiol 1992;45:613-619.

14 Sohn MW, Arnold N, Maynard C, Hynes DM: Accuracy and completeness of mortality data in the Department of Veterans Affairs. Popul Health Metr 2006;4:2.

15 Moist LM, Lok CE, Vachharajani TJ, Xi W, AlJaishi A, Polkinghorne KR, Vazquez M, Lee TC: Optimal hemodialysis vascular access in the elderly patient. Semin Dial 2012;25:640648.

16 O'Hare AM: Vascular access for hemodialysis in older adults: a "patient first" approach. J Am Soc Nephrol 2013;24:1187-1190.

17 Ethier J, Mendelssohn DC, Elder SJ, Hasegawa T, Akizawa T, Akiba T, Canaud BJ, Pisoni RL: Vascular access use and outcomes: an international perspective from the Dialysis Outcomes and Practice Patterns Study. Nephrol Dial Transplant 2008;23:3219-3226.
18 Staramos DN, Lazarides MK, Tzilalis VD, Ekonomou CS, Simopoulos CE, Dayantas JN: Patency of autologous and prosthetic arteriovenous fistulas in elderly patients. Eur J Surg 2000; 166:777-781.

19 Berardinelli L, Vegeto A: Lessons from 494 permanent accesses in 348 haemodialysis patients older than 65 years of age: 29 years of experience. Nephrol Dial Transplant 1998; 13(suppl 7):73-77.

20 Allon M, Lok CE: Dialysis fistula or graft: the role for randomized clinical trials. Clin J Am Soc Nephrol 2010;5:2348-2354.

21 Schild AF, Perez E, Gillaspie E, Seaver C, Livingstone J, Thibonnier A: Arteriovenous fistulae vs. arteriovenous grafts: a retrospective review of 1,700 consecutive vascular access cases. J Vasc Access 2008;9:231235.

22 Chan MR, Sanchez RJ, Young HN, Yevzlin AS: Vascular access outcomes in the elderly hemodialysis population: a USRDS study. Semin Dial 2007;20:606-610.

23 DeSilva RN, Patibandla BK, Vin Y, Narra A, Chawla V, Brown RS, Goldfarb-Rumyantzev AS: Fistula first is not always the best strategy for the elderly. J Am Soc Nephrol 2013;24: 1297-1304.

24 Quinn RR, Oliver MJ, Devoe D, Poinen K, Kabani R, Kamar F, Mysore P, Lewin AM, Hiremath S, MacRae J, James MT, Miller L, Hemmelgarn BR, Moist LM, Garg AX, Chowdhury TT, Ravani P: The effect of predialysis fistula attempt on risk of all-cause and access-related death. J Am Soc Nephrol 2017;28:613-620.

25 Brown RS, Patibandla BK, Goldfarb-Rumyantzev AS: The survival benefit of "fistula first, catheter last" in hemodialysis is primarily due to patient factors. J Am Soc Nephrol 2017;28: 645-652.

26 Lorenzo V, Martn M, Rufino M, Hernandez D, Torres A, Ayus JC: Predialysis nephrologic care and a functioning arteriovenous fistula at entry are associated with better survival in incident hemodialysis patients: an observational cohort study. Am J Kidney Dis 2004;43: 999-1007. 